\title{
Analysis of minimum zone sphericity error using minimum potential energy theory
}

\author{
Kuang-Chao Fan ${ }^{\mathrm{a}, *}, \mathrm{Ji}^{-C h u n}$ Lee $^{\mathrm{b}}$ \\ ${ }^{a}$ Department of Mechanical Engineering, National Taiwan University, Taipei, Taiwan, Republic of China \\ ${ }^{\mathrm{b}}$ Department of Mechanical Engineering, National Chung-Hsing University, Taipei, Taiwan, Republic of China
}

Received 28 October 1997; received in revised form 2 June 1998; accepted 28 August 1998

\begin{abstract}
Theoretical derivation of the minimum zone criteria of sphericity error based on the principle of minimum potential energy is proposed. All the measured data points are enclosed by two concentric spherical surfaces between which a fictitious spring is assumed to be placed. These two concentric spherical surfaces can be mathematically determined by five active data points. When the spring contracts, the potential energy of the simulated mechanical system tends to reduce which yields two new concentric spheres with smaller radial separation and new active data points. Finally, a stable state will be reached to the condition of minimum potential energy. The criteria conforming to such a state can be derived. A direct search scheme to the global minimum solution is also proposed. The clearance between such two concentric spherical surfaces is the minimum zone of spherical form error. (c) 1999 Elsevier Science Inc. All rights reserved.
\end{abstract}

Keywords: Sphericity error; Minimum zone method; Potential energy; Virtual work; Static equilibrium

\section{Introduction}

Sphericity is a kind of form error which is broadly used in industry for the geometrical measurement of precision balls. Any defects on the surface, such as surface roughness and form error, may result in the life reduction, wear, and run-out rotation. Therefore, effective and precise evaluation of the sphericity is an important issue.

There are various kinds of form errors, such as straightness, flatness, roundness, sphericity, and cylindricity. There are also many kinds of evaluation techniques. Cheraghi [1] formulated the straightness and flatness errors by nonlinear optimization problems with a linear objective function and nonlinear constraints. Wang [2] applied the theory of nonlinear constrained optimization to evaluate straightness, flatness, roundness, and cylindricity. Kanada [3] computed the minimum zone sphericity using iterative least squares and the downhill simplex methods. Carr [4,5] proposed minimum zone searching algorithms that solved a sequence of linear problems and converged to the solution of the nonlinear one. Huang [6,7] evaluated the minimum zone

$$
1186 .
$$

* Corresponding author. Tel.: +886-2-2362-0032; fax: +886-2-2364-

E-mail address: fan@ccms.ntu.edu.tw (K.-C. Fan) straightness and flatness form error based on the control line, or control plane, rotation scheme (CLRS or CPRS) which could result in the exact solutions. In this article, we have not only derived some lemmas for finding the criteria of minimum zone sphericity error, but also proposed a fast computational algorithm, starting from the least squares result, which can direct search to the minimum zone state.

\section{Mechanical model of minimum zone spherical form error}

A simulated mechanical system is used to model the problem of minimum zone spherical form error. A group of supports are fixed at positions of measured data points and enclosed by two fictitious concentric semi-rigid spherical surfaces. These supports are assumed to withstand compression only. The concentric semi-rigid spherical surfaces should observe the law that they remain concentric and spherical when deformed. A fictitious spring is assumed to be placed between them, as shown in Fig. 1, and is the only component of the mechanical system that can store the elastic energy.

From the geometrical point of view, the degree of freedom of the system is five and there are five active supports 


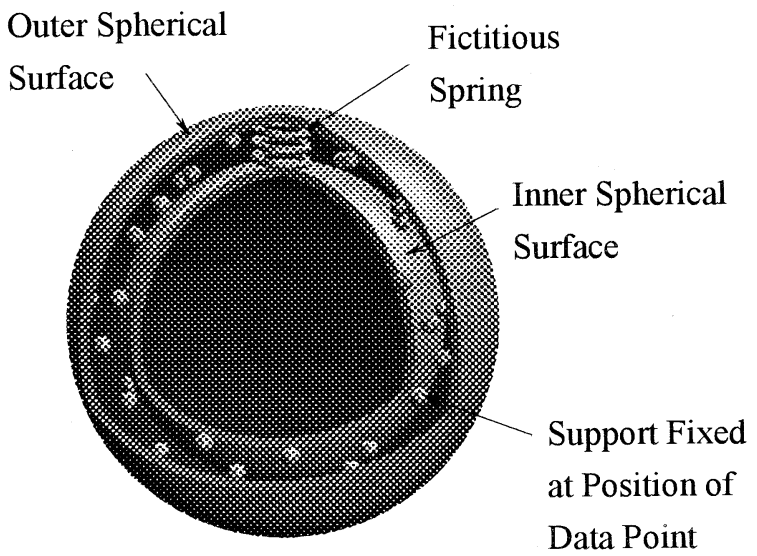

Fig. 1. Mechanical model of minimum zone spherical form error problem.

at the same time. These five active supports are located at the corresponding five measured points which are contacted by the enclosing spherical surfaces. In other words, at least five points must be in contact with the two enclosing concentric spherical surfaces. When the spring contracts, these active supports will be replaced by new contact points and the elastic energy of the system will tend to reduce. Finally, it will reach a stable state of minimum potential energy. The problem of the minimum zone spherical form error is then transformed into the problem of minimum potential energy of the simulated mechanical system. It can be stated as: Minimizing the elastic potential energy

$U=\frac{1}{2} k u^{2}$

subjected to all supports (measured data points) enclosed by the two concentric spherical surfaces. In Eq. (1), $k$ is the spring constant of the fictitious spring and $u$ is its displacement.

\section{Formulation of system equations}

From the geometrical point of view, there are four modes of concentric spherical surfaces: (1) Mode M4-1, four data points on the outer sphere and the last data point on the inner sphere; (2) mode M3-2, three data points on the outer sphere and the other two data points on the inner sphere; (3) mode M2-3, two data points on the outer sphere and the other three data points on the inner sphere; (4) Mode M1-4, one data point on the outer sphere and the other four data points on the inner sphere. Assume the current active data points are $P_{1}$ to $P_{5}$ and the active supports of the simulated mechanical system are placed at these five positions respectively. When these supports are released the free body diagrams are shown in Fig. 2 in which reactions on the active supports of $P_{1}$ to $P_{5}$ are $N_{1}$ to $N_{5}$ respectively, and $P_{i}$ is the corresponding point on the outer surface when $P_{i}$ is on the inner surface. Without the loss of generality, let the center of the concentric sphere be the origin of the coordinate system, the outer sphere be the unit sphere (radius $\left.R_{\mathrm{O}}=1\right)$, and the radius of the inner sphere be $R_{i}\left(R_{i}=R_{o} / \alpha\right.$ $=1 / \alpha, \alpha>1)$. Let the coordinates of these active data points be $P_{1}\left(x_{1}, y_{1}, z_{1}\right), P_{2}\left(x_{2}, y_{2}, z_{2}\right), P_{3}\left(x_{3}, y_{3}, z_{3}\right), P_{4}\left(x_{4}, y_{4}, z_{4}\right)$ and $P_{5}\left(x_{5}, y_{5}, z_{5}\right)$. For mode M4-1 the equilibrium equations of reactions are:

$$
\begin{aligned}
& \sum F_{x}=0 \\
& N_{1} x_{1}+N_{2} x_{2}+N_{3} x_{3}+N_{4} x_{4}-N_{5} \alpha x_{5}=0 \\
& \sum F_{y}=0 \\
& N_{1} y_{1}+N_{2} y_{2}+N_{3} y_{3}+N_{4} y_{4}-N_{5} \alpha y_{5}=0 \\
& \sum F_{z}=0 \\
& N_{1} z_{1}+N_{2} z_{2}+N_{3} z_{3}+N_{4} z_{4}-N_{5} \alpha z_{5}=0
\end{aligned}
$$

where $\Sigma F_{x}=0, \Sigma F_{y}=0, \Sigma F_{z}=0$ mean the sum of the $\mathrm{x}, \mathrm{y}$, and $\mathrm{z}$ components of all reactions should equal to zero at equilibrium condition.

$$
\text { Let [Eq. (5)] }
$$

$$
P^{\prime}{ }_{5}=\left(x^{\prime}{ }_{5}, y^{\prime}{ }_{5}, z^{\prime}{ }_{5}\right)=\alpha\left(x_{5}, y_{5}, z_{5}\right)
$$

The corresponding data point $P^{\prime}{ }_{5}\left(x^{\prime}{ }_{5}, y^{\prime}{ }_{5}, z^{\prime}{ }_{5}\right)$ with respect to $P_{5}$ is on the outer unit sphere (shown in Fig. 2a). Assume the center of these two concentric spheres and the inner spherical surface are fixed and the outer spherical surface is given an admissible virtual displacement $\delta u$. From the principle of virtual work, we have

$$
\begin{aligned}
& N_{1} \delta u+N_{2} \delta u+N_{3} \delta u+N_{4} \delta u=\delta\left(\frac{1}{2} k u^{2}\right)=k u \delta u \\
& N_{1}+N_{2}+N_{3}+N_{4}=k u=C \geq 0
\end{aligned}
$$

Similarly, Let the center of these concentric spheres and the outer spherical surface be fixed and the inner spherical surface be given an admissible virtual displacement $\delta \mathrm{u}$. We have

$$
\begin{aligned}
& N_{5} \delta u=\delta\left(\frac{1}{2} k u^{2}\right)=k u \delta u \\
& N_{5}=k u=C \geq 0
\end{aligned}
$$

Combining Eq.(2) to (4) and (6), (7), the system equations of mode M4-1 can be represented in matrix form as [Eq. (8)]

$$
\left[\begin{array}{ccccc}
x_{1} & x_{2} & x_{3} & x_{4} & -x^{\prime}{ }_{5} \\
y_{1} & y_{2} & y_{3} & y_{4} & -y^{\prime}{ }_{5} \\
z_{1} & z_{2} & z_{3} & z_{4} & -z^{\prime}{ }_{5} \\
1 & 1 & 1 & 1 & 0 \\
0 & 0 & 0 & 0 & 1
\end{array}\right]\left\{\begin{array}{l}
N_{1} \\
N_{2} \\
N_{3} \\
N_{4} \\
N_{5}
\end{array}\right\}=\left\{\begin{array}{l}
0 \\
0 \\
0 \\
C \\
C
\end{array}\right\}
$$

Similarly, from equilibrium equations and the principle of virtual work we have the system equations of mode M3-2 as [Eq. (9)] 


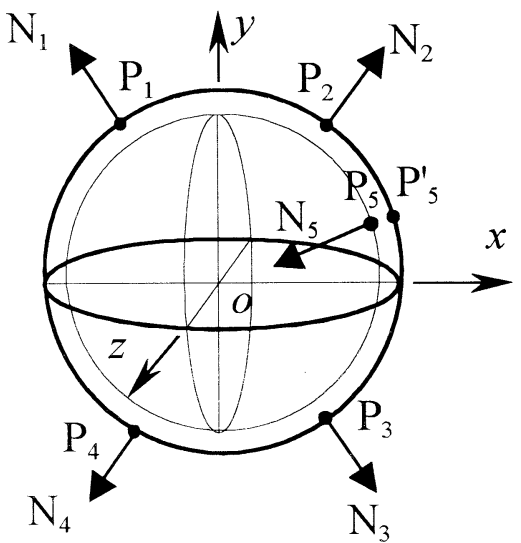

(a)

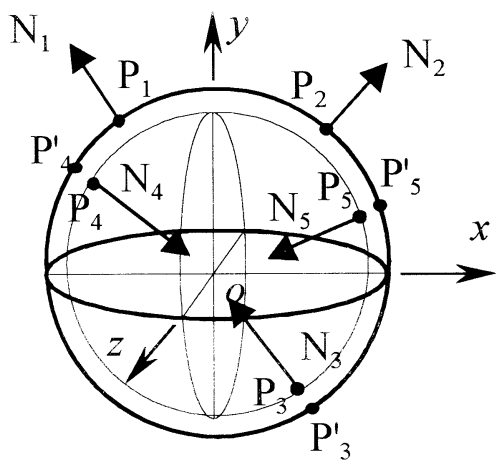

(c)

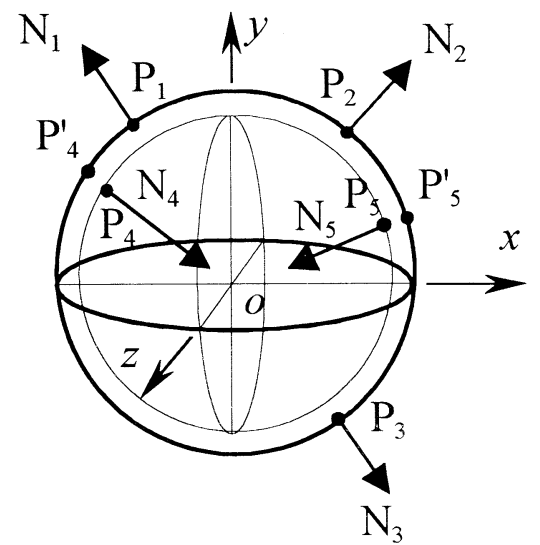

(b)

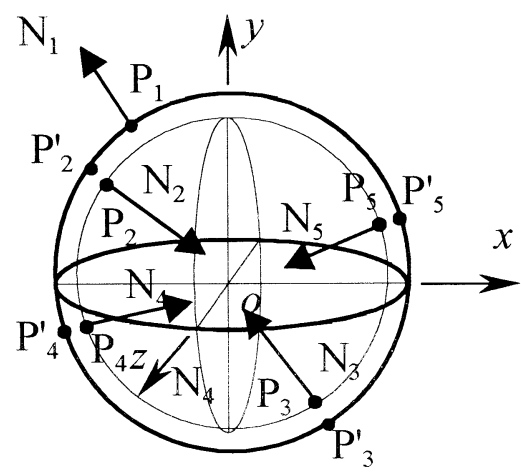

(d)

Fig. 2. Free body diagrams of four modes of concentric spherical surfaces: a) Mode M4-1, b) Mode M3-2, c) Mode M2-3, d) Mode M1-4.

$$
\left[\begin{array}{ccccc}
x_{1} & x_{2} & x_{3} & -x^{\prime}{ }_{4} & -x^{\prime}{ }_{5} \\
y_{1} & y_{2} & y_{3} & -y^{\prime}{ }_{4} & -y^{\prime}{ }_{5} \\
z_{1} & z_{2} & z_{3} & -z^{\prime}{ }_{4} & -z^{\prime}{ }_{5} \\
1 & 1 & 1 & 0 & 0 \\
0 & 0 & 0 & 1 & 1
\end{array}\right]\left\{\begin{array}{l}
N_{1} \\
N_{2} \\
N_{3} \\
N_{4} \\
N_{5}
\end{array}\right\}=\left\{\begin{array}{l}
0 \\
0 \\
0 \\
C \\
C
\end{array}\right\}
$$

The system equations of mode M2-3 are [Eq. (10)]

$$
\left[\begin{array}{ccccc}
x_{1} & x_{2}-x_{3}^{\prime} & -x^{\prime}{ }_{4} & -x^{\prime}{ }_{5} \\
y_{1} & y_{2} & -y^{\prime}{ }_{3} & -y^{\prime}{ }_{4} & -y^{\prime}{ }_{5} \\
z_{1} & z_{2} & -z^{\prime}{ }_{3} & -z^{\prime}{ }_{4} & -z^{\prime}{ }_{5} \\
1 & 1 & 0 & 0 & 0 \\
0 & 0 & 1 & 1 & 1
\end{array}\right]\left\{\begin{array}{l}
N_{1} \\
N_{2} \\
N_{3} \\
N_{4} \\
N_{5}
\end{array}\right\}=\left\{\begin{array}{l}
0 \\
0 \\
0 \\
C \\
C
\end{array}\right\}
$$

The system equations of mode M1-4 are [Eq. (11)]

$$
\left[\begin{array}{ccccc}
x_{1}-x_{2}^{\prime} & -x_{3}^{\prime} & -x^{\prime}{ }_{4} & -x^{\prime}{ }_{5} \\
y_{1} & -y_{2}^{\prime}{ }_{2} & -y_{3}^{\prime} & -y^{\prime}{ }_{4} & -y^{\prime}{ }_{5} \\
z_{1} & -z_{2}^{\prime} & -z_{3}^{\prime} & -z_{4}^{\prime} & -z^{\prime}{ }_{5} \\
1 & 0 & 0 & 0 & 0 \\
0 & 1 & 1 & 1 & 1
\end{array}\right]\left\{\begin{array}{l}
N_{1} \\
N_{2} \\
N_{3} \\
N_{4} \\
N_{5}
\end{array}\right\}=\left\{\begin{array}{l}
0 \\
0 \\
0 \\
C \\
C
\end{array}\right\}
$$

Solving the system equations for each mode, we obtain the corresponding reactions on each support for each mode.

\section{Theorem of minimum zone spherical form error}

In a stable state, the reactions at all active supports must be compression. If the reactions are tension then the spherical 

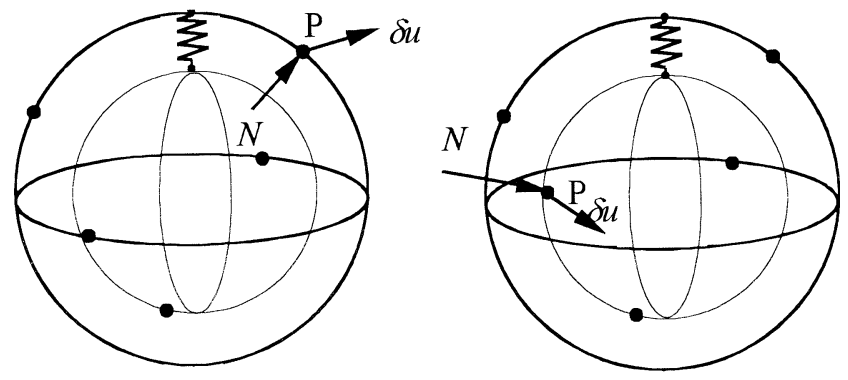

Fig. 3. Free body diagram with one active support released.

surface and the support will separate and the system will not be stable. In this article, the sign convention of the reaction is positive for compression, and negative for tension. In other words, for reactions that act on the outer spherical surface with direction outward are positive and reactions that act on the inner spherical surface with direction inward are positive. It can be shown that when all the active reactions are positive (i.e., compression), the simulated mechanical system is in a stable state and has minimum elastic potential energy.

Lemma 1: The simulated mechanical system is in a stable state if all reactions at active supports are positive.

Proof:

Let the support at data point $\mathrm{P}$ on the concentric spherical surfaces be released and replaced by the reaction $N$ (shown in Fig. 3) and all other supports remain unchanged.

By Castigliano's first theorem [8] we have [Eq. (12)]

$\delta U=\vec{N} \cdot \delta \vec{u}>0$

where $\delta U$ is the change of potential energy of the system due to the displacement $\delta u$ of the outer spherical surface at $P$. For a stable system, the change of potential energy of the system must be positive.

Because all supports must be enclosed by the two concentric spherical surfaces. The direction of admissible displacement $\delta \vec{u}$ must be outward if the released active support is located at the outer spherical surface, and inward if it is located at the inner spherical surface. From Eq. (12), we can also conclude that reaction $N$ and displacement $\delta u$ must have the same direction (i.e., reaction $N$ is positive). According to the above discussion, we reach the conclusion that when all reactions are positive (compression), any further deformation of the two concentric spherical surfaces will lead to the increase of elastic potential energy of the system. In other words, if all reactions are positive the system is then in a stable state.

Lemma 2: Mode M4-1 and M1-4 are unstable.

\section{Proof:}

Let $\mathrm{C}=1$ for convenience, then the system equations of mode M4-1 are [Eq. (13)]

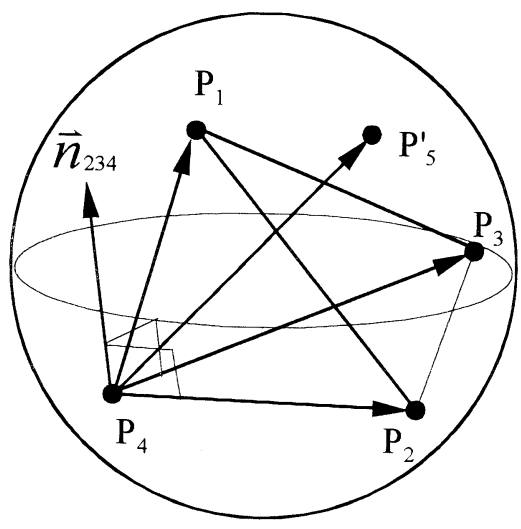

Fig. 4. Schematic diagram of Mode M4-1.

$$
\left[\begin{array}{ccccc}
x_{1} & x_{2} & x_{3} & x_{4} & -x^{\prime}{ }_{5} \\
y_{1} & y_{2} & y_{3} & y_{4} & -y^{\prime}{ }_{5} \\
z_{1} & z_{2} & z_{3} & z_{4} & -z^{\prime}{ }_{5} \\
1 & 1 & 1 & 1 & 0 \\
0 & 0 & 0 & 0 & 1
\end{array}\right]\left\{\begin{array}{l}
N_{1} \\
N_{2} \\
N_{3} \\
N_{4} \\
N_{5}
\end{array}\right\}=\left\{\begin{array}{l}
0 \\
0 \\
0 \\
1 \\
1
\end{array}\right\}
$$

From Equation (13), we have $\mathrm{N}_{5}=1$, and this equation can be simplified to [Eq. (14)]

$$
\left[\begin{array}{cccc}
x_{1} & x_{2} & x_{3} & x_{4} \\
y_{1} & y_{2} & y_{3} & y_{4} \\
z_{1} & z_{2} & z_{3} & z_{4} \\
1 & 1 & 1 & 1
\end{array}\right]\left\{\begin{array}{l}
N_{1} \\
N_{2} \\
N_{3} \\
N_{4}
\end{array}\right\}=\left\{\begin{array}{c}
x_{5}^{\prime} \\
y_{5}^{\prime} \\
z_{5}^{\prime} \\
1
\end{array}\right\}
$$

Let

$$
D=\left[\begin{array}{cccc}
x_{1} & x_{2} & x_{3} & x_{4} \\
y_{1} & y_{2} & y_{3} & y_{4} \\
z_{1} & z_{2} & z_{3} & z_{4} \\
1 & 1 & 1 & 1
\end{array}\right] \quad D_{1}=\left[\begin{array}{cccc}
x^{\prime} & x_{2} & x_{3} & x_{4} \\
y^{\prime} & y_{2} & y_{3} & y_{4} \\
z^{\prime}{ }_{5} & z_{2} & z_{3} & z_{4} \\
1 & 1 & 1 & 1
\end{array}\right]
$$

Expressing in vector form, Equation (15) becomes [Eq. (16)]

$D=\vec{r}_{14} \cdot \vec{n}_{234} \quad$ and $\quad D_{1}=\vec{r}_{54} \cdot \vec{n}_{234}$

where

$\vec{r}_{i}=\left(x_{i}, y_{i}, z_{i}\right)$ or $\left(x_{i}^{\prime}, y_{i}^{\prime}, z_{i}^{\prime}\right)$

$\vec{r}_{i j}=\vec{r}_{i}-\vec{r}_{j}$

$\vec{n}_{234}=\vec{r}_{24} \times \vec{r}_{34}$

and the last term of above refer to the normal of the plane formed by points $\mathrm{P}_{2}, \mathrm{P}_{3}$, and $\mathrm{P}_{4}$. From the Cramer's rule, we have [Eq. (17)]

$N_{1}=\frac{D_{1}}{D}=\frac{\vec{r}_{54} \cdot \vec{n}_{234}}{\vec{r}_{14} \cdot \vec{n}_{234}}$ 
From Equation (17), we conclude that the sufficient and necessary condition for $N_{1}>0$ is [Eq. (18)]

$\vec{r}_{14} \cdot \vec{n}_{234}>0$ and $\vec{r}_{54} \cdot \vec{n}_{234}>0$

or $\vec{r}_{14} \cdot \vec{n}_{234}<0$ and $\vec{r}_{54} \cdot \vec{n}_{234}<0$

This implies that $\mathrm{P}_{1}$ and $\mathrm{P}_{5}^{\prime}$ should be on the same side of the plane formed by $\mathrm{P}_{2}, \mathrm{P}_{3}, \mathrm{P}_{4}$ (shown in Fig. 4). Similarly, we can also conclude that [Equations (19), (20), (21)]

$\vec{r}_{24} \cdot \vec{n}_{314}>0, \vec{r}_{54} \cdot \vec{n}_{314}>$ or $\vec{r}_{24} \cdot \vec{n}_{314}<0$,

$\vec{r}_{54} \cdot \vec{n}_{314}<0$

$\vec{r}_{34} \cdot \vec{n}_{124}>0, \vec{r}_{54} \cdot \vec{n}_{124}>$ or $\vec{r}_{34} \cdot \vec{n}_{124}<0$,

$\vec{r}_{54} \cdot \vec{n}_{124}<0$

$\vec{r}_{41} \cdot \vec{n}_{321}>0, \vec{r}_{51} \cdot \vec{n}_{321}>$ or $\vec{r}_{41} \cdot \vec{n}_{321}<0$,

$\vec{r}_{51} \cdot \vec{n}_{321}<0$

According to the above relations, the necessary and sufficient condition for $\mathrm{N}_{2}>0$ is that $\mathrm{P}_{2}$ and $\mathrm{P}^{\prime}{ }_{5}$ should be on the same side of the plane formed by $\mathrm{P}_{1}, \mathrm{P}_{3}, \mathrm{P}_{4}$; for $\mathrm{N}_{3}>0, \mathrm{P}_{3}$ and $\mathrm{P}^{\prime}{ }_{5}$ should be on the same side of the plane formed by $\mathrm{P}_{1}, \mathrm{P}_{2}, \mathrm{P}_{4}$; and for $\mathrm{N}_{4}>0, \mathrm{P}_{4}$ and $\mathrm{P}^{\prime}{ }_{5}$ should be on the same side of the plane formed by $\mathrm{P}_{1}, \mathrm{P}_{2}, \mathrm{P}_{3}$. We then have the following conclusion: the necessary and sufficient condition for all reactions to be positive (compression) is that the point $\mathrm{P}^{\prime}{ }_{5}$ should be located inside the tetrahedron formed by $\mathrm{P}_{1}, \mathrm{P}_{2}, \mathrm{P}_{3}, \mathrm{P}_{4}$. However, since $\mathrm{P}_{1}, \mathrm{P}_{2}, \mathrm{P}_{3}, \mathrm{P}_{4}, \mathrm{P}^{\prime}{ }_{5}$ are all on the same unit sphere, the conclusion made above is impossible. In other words, Modes M4-1 is unstable. This situation can also be applied to mode M1-4.

Lemma 3: Mode M3-2 is stable if and only if $\mathrm{P}_{4}{ }_{4}$ and $\mathrm{P}^{\prime}{ }_{5}$ are on the opposite sides of the plane formed by $\mathrm{P}_{1}, \mathrm{P}_{2}, \mathrm{P}_{3}$ and the line segment $\mathrm{P}_{4}^{\prime} \mathrm{P}^{\prime}{ }_{5}$ intersects the triangle $\Delta \mathrm{P}_{1}, \mathrm{P}_{2}, \mathrm{P}_{3}$; Mode M2-3 is stable if and only if $\mathrm{P}_{1}$ and $\mathrm{P}_{2}$ are on the opposite sides of the plane formed by $\mathrm{P}_{3}{ }_{3}, \mathrm{P}_{4}^{\prime}, \mathrm{P}^{\prime}{ }_{5}$ and the line segment $\mathrm{P}_{1} \mathrm{P}_{2}$ intersects the triangle formed by $\Delta \mathrm{P}_{3}^{\prime}, \mathrm{P}_{4}^{\prime}, \mathrm{P}^{\prime}{ }_{5}$.

Proof:

Let $\mathrm{C}=1$, then the system equations of mode M3-2 are

$\left[\begin{array}{ccccc}x_{1} & x_{2} & x_{3} & -x_{4}^{\prime} & -x^{\prime}{ }_{5} \\ y_{1} & y_{2} & y_{3} & -y^{\prime}{ }_{4} & -y^{\prime}{ }_{5} \\ z_{1} & z_{2} & z_{3} & -z_{4_{4}}^{\prime} & -z^{\prime}{ }_{5} \\ 1 & 1 & 1 & 0 & 0 \\ 0 & 0 & 0 & 1 & 1\end{array}\right]\left\{\begin{array}{l}N_{1} \\ N_{2} \\ N_{3} \\ N_{4} \\ N_{5}\end{array}\right\}=\left\{\begin{array}{l}0 \\ 0 \\ 0 \\ 1 \\ 1\end{array}\right\}$

We have

$N_{4}+N_{5}=1$

$N_{5}=1-N_{4}$
Substituting Equation (23) into Equation (22) yields: [Eq. (24)]

$\left[\begin{array}{cccc}x_{1} & x_{2} & x_{3} & x^{\prime}{ }_{5}-x^{\prime}{ }_{4} \\ y_{1} & y_{2} & y_{3} & y^{\prime}{ }_{5}-y^{\prime}{ }_{4} \\ z_{1} & z_{2} & z_{3} & z^{\prime}{ }_{5}-z^{\prime}{ }_{4} \\ 1 & 1 & 1 & 0\end{array}\right]\left\{\begin{array}{c}N_{1} \\ N_{2} \\ N_{3} \\ N_{4}\end{array}\right\}=\left\{\begin{array}{c}x^{\prime}{ }_{5} \\ y^{\prime}{ }_{5} \\ z^{\prime}{ }_{5} \\ 1\end{array}\right\}$

Let [Eq. (25)]

$D=\left[\begin{array}{cccc}x_{1} & x_{2} & x_{3} & x^{\prime}{ }_{5}-x^{\prime}{ }_{4} \\ y_{1} & y_{2} & y_{3} & y^{\prime}{ }_{5}-y_{4}^{\prime} \\ z_{1} & z_{2} & z_{3} & z^{\prime}{ }_{5}-z^{\prime}{ }_{4} \\ 1 & 1 & 1 & 0\end{array}\right]$,

$D_{1}=\left[\begin{array}{cccc}x^{\prime} & x_{2} & x_{3} & x^{\prime}{ }_{5}-x^{\prime}{ }_{4} \\ y^{\prime}{ }_{5} & y_{2} & y_{3} & y^{\prime}{ }_{5}-y^{\prime}{ }_{4} \\ z^{\prime}{ }_{5} & z_{2} & z_{3} & z^{\prime}{ }_{5}-z^{\prime}{ }_{4} \\ 1 & 1 & 1 & 0\end{array}\right]$,

$D_{2}=\left[\begin{array}{cccc}x_{1} & x^{\prime}{ }_{5} & x_{3} & x^{\prime}{ }_{5}-x^{\prime}{ }_{4} \\ y_{1} & y^{\prime}{ }_{5} & y_{3} & y^{\prime}{ }_{5}-y^{\prime}{ }_{4} \\ z_{1} & z^{\prime}{ }_{5} & z_{3} & z^{\prime}{ }_{5}-z^{\prime}{ }_{4} \\ 1 & 1 & 1 & 0\end{array}\right]$

$D_{3}=\left[\begin{array}{cccc}x_{1} & x_{2} & x^{\prime}{ }_{5} & x^{\prime}{ }_{5}-x^{\prime}{ }_{4} \\ y_{1} & y_{2} & y^{\prime}{ }_{5} & y^{\prime}{ }_{5}-y^{\prime}{ }_{4} \\ z_{1} & z_{2} & z^{\prime}{ }_{5} & z^{\prime}{ }_{5}-z^{\prime}{ }_{4} \\ 1 & 1 & 1 & 0\end{array}\right], \quad D_{4}=\left[\begin{array}{cccc}x_{1} & x_{2} & x_{3} & x^{\prime}{ }_{5} \\ y_{1} & y_{2} & y_{3} & y^{\prime}{ }_{5} \\ z_{1} & z_{2} & z_{3} & z^{\prime}{ }_{5} \\ 1 & 1 & 1 & 1\end{array}\right]$

Expressing in vector form, Equations (25) become [Eq. (26)]

$D=-\vec{r}_{54} \cdot \vec{n}_{231}$

$D_{1}=-\vec{r}_{54} \cdot \vec{n}_{235}$

$D_{2}=-\vec{r}_{54} \cdot \vec{n}_{315}$

$D_{3}=-\vec{r}_{54} \cdot \vec{n}_{125}$

$D_{4}=-\vec{r}_{51} \cdot \vec{n}_{231}$

where

$\vec{r}_{i}=\left(x_{i}, y_{i}, z_{i}\right)$ or $\left(x_{i}^{\prime}, y_{i}^{\prime}, z_{i}^{\prime}\right)$

$\vec{r}_{i j}=\vec{r}_{i}-\vec{r}_{j}$

$r n_{i j k}=\vec{r}_{i k} \times \vec{r}_{j k}=\left(\vec{r}-\vec{r}_{k}\right) \times\left(\vec{r}_{j}-\vec{r}_{k}\right)$

From the Cramer's rule, we have [Equations (27), (28), (29), (30)] 


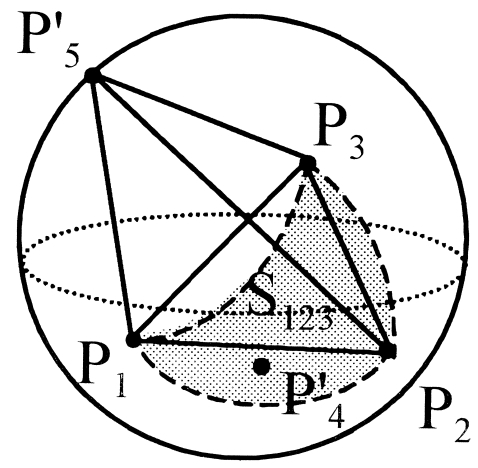

Fig. 5. Solution of case I of Mode M3-2.

$N_{1}=\frac{D_{1}}{D}=\frac{\vec{r}_{54} \cdot \vec{n}_{235}}{\vec{r}_{54} \cdot \vec{n}_{231}}, \quad N_{2}=\frac{D_{2}}{D}=\frac{\vec{r}_{54} \cdot \vec{n}_{315}}{\vec{r}_{54} \cdot \vec{n}_{231}}$

$N_{3}=\frac{D_{3}}{D}=\frac{\vec{r}_{54} \cdot \vec{n}_{125}}{\vec{r}_{54} \cdot \vec{n}_{231}}, \quad N_{4}=\frac{D_{4}}{D}=\frac{\vec{r}_{51} \cdot \vec{n}_{231}}{\vec{r} l_{54} \cdot \vec{n}_{231}}$

Case I: When $\vec{r}_{54} \cdot \vec{n}_{231}>0$

$N_{1}>0$ if and only if $\vec{r}_{54} \cdot \vec{n}_{235}>0$

$N_{2}>0$ if and only if $\vec{r}_{54} \cdot \vec{n}_{315}>0$

$N_{3}>0$ if and only if $\vec{r}_{54} \cdot \vec{n}_{125}>0$

$N_{4}>0$ if and only if $\vec{r}_{51} \cdot \vec{n}_{231}>0$

From Eqs. (28) to (31), we conclude that $\mathrm{P}^{\prime}{ }_{4}$ should locate on the spherical surface $\mathrm{S}_{123}$ as shown in Fig. 5 .

Hence,

$\vec{r}_{54} \cdot \vec{n}_{231}>\vec{r}_{51} \cdot \vec{n}_{231}>0$

$1>N_{4}>0$

From Equation (23), we have [Equations (32), (33), (34), (35)]

$N_{5}=1-N_{4}>0$
Case II. When $\vec{r}_{54} \cdot \vec{n}_{231}<0$

$N_{1}>0$ if and only if $\vec{r}_{54} \cdot \vec{n}_{235}<0$

$N_{2}>0$ if and only if $\vec{r}_{54} \cdot \vec{n}_{315}<0$

$N_{3}>0$ if and only if $\vec{r}_{54} \cdot \vec{n}_{125}<0$

$N_{4}>0$ if and only if $\vec{r}_{51} \cdot \vec{n}_{231}<0$

In this case, the solution that satisfies Eqs. (32) to (35) does not exist.

From the above discussions, we can see that mode M3-2 is stable, and all reactions are positive if and only if $\mathrm{P}_{4}$ and $\mathrm{P}^{\prime}{ }_{5}$ are on the opposite sides of the plane formed by $\mathrm{P}_{1}, \mathrm{P}_{2}, \mathrm{P}_{3}$, and the line segment $\mathrm{P}^{\prime}{ }_{4} \mathrm{P}^{\prime}$ intersects the triangle $\Delta \mathrm{P}_{1} \mathrm{P}_{2} \mathrm{P}_{3}$. Similarly, mode $\mathrm{M} 2-3$ is stable if and only if $\mathrm{P}_{1}$ and $\mathrm{P}_{2}$ are on the opposite sides of the plane formed by $\mathrm{P}_{3}^{\prime}, \mathrm{P}^{\prime}{ }_{4}, \mathrm{P}^{\prime}{ }_{5}$ and the line segment $\mathrm{P}_{1} \mathrm{P}_{2}$ intersects the triangle $\Delta \mathrm{P}_{3}^{\prime} \mathrm{P}_{4}^{\prime} \mathrm{P}^{\prime}{ }_{5}$. These are the criteria for the minimum zone condition of sphericity error.

\section{Computational algorithm}

Let's call those five data points that determine the concentric spheres are active data points contained in the set $\mathrm{S}$. From Lemma 1, we know that the reactions act on the active points should be positive. If any of these reactions is negative, it means that the potential energy can be further reduced. In the other words, the data point of negative reaction can be removed from the set $\mathrm{S}$ and new searched data point should be added in such that they can form two new concentric spheres having less potential energy. In general, there may be more than one negative reaction in the beginning of the search process. In this computational algorithm, we only exchange one active point each time and the data point which has the most negative reaction is chosen to be removed from $\mathrm{S}$. This state is called the

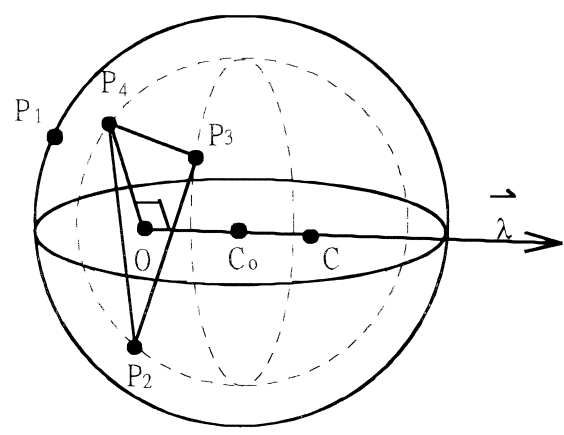

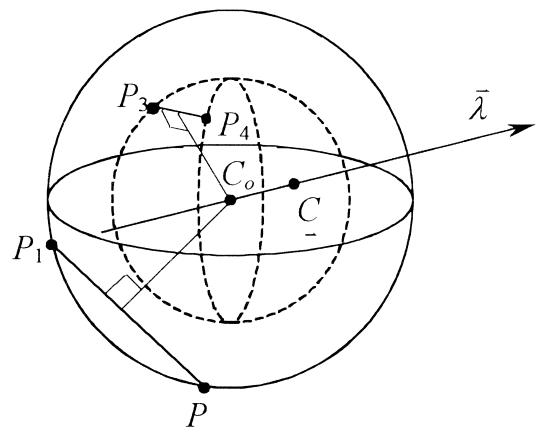

(a)

(b)

Fig. 6. Generating new search direction: a) for Mode T1-3, b) for mode T2-2. 
Table 1

Sphericity data set and its result (unit: $\mathrm{mm}$ )

\begin{tabular}{|c|c|c|c|}
\hline No. & $\mathrm{X}$ & $\mathrm{Y}$ & $\mathrm{Z}$ \\
\hline 1 & 0.81814 & -0.43615 & -0.38525 \\
\hline 2 & -0.80885 & -0.53045 & 0.27905 \\
\hline 3 & 0.03749 & 0.90643 & -0.42737 \\
\hline 4 & 0.86935 & -0.27403 & -0.42518 \\
\hline 5 & -0.50786 & -0.07441 & 0.86060 \\
\hline 6 & 0.75348 & -0.67012 & -0.00610 \\
\hline 7 & 0.05867 & -1.00773 & -0.00198 \\
\hline 8 & -0.17486 & 0.60442 & 0.78297 \\
\hline 9 & 0.71121 & -0.50123 & -0.50042 \\
\hline 10 & -0.47493 & -0.51466 & 0.71992 \\
\hline 11 & 0.06016 & -0.74129 & -0.67432 \\
\hline 12 & 0.05337 & 0.02006 & 1.00515 \\
\hline 13 & 0.29869 & 0.87920 & 0.38988 \\
\hline 14 & -0.15488 & -0.86697 & 0.48585 \\
\hline 15 & 0.38994 & -0.00060 & -0.92592 \\
\hline 16 & 0.00776 & 0.73922 & 0.68741 \\
\hline 17 & 0.62090 & 0.52006 & -0.59341 \\
\hline 18 & 0.00952 & -0.03493 & -1.00376 \\
\hline 19 & -0.44592 & 0.76584 & -0.47827 \\
\hline 20 & 0.01069 & 0.99511 & 0.11265 \\
\hline 21 & -0.93371 & -0.17351 & -0.33160 \\
\hline 22 & -0.48121 & -0.69836 & 0.53717 \\
\hline 23 & 0.50394 & 0.86933 & 0.03322 \\
\hline 24 & 0.80924 & 0.07332 & -0.59398 \\
\hline 25 & 0.84569 & 0.06876 & -0.53570 \\
\hline 26 & 0.36179 & -0.07902 & 0.93410 \\
\hline 27 & -0.78624 & 0.20326 & -0.59063 \\
\hline 28 & -0.46529 & 0.69616 & -0.55167 \\
\hline 29 & -0.93402 & -0.02267 & -0.35813 \\
\hline 30 & 0.43016 & 0.49319 & -0.76122 \\
\hline 31 & -0.46929 & 0.11501 & 0.88119 \\
\hline 32 & 0.60788 & -0.41130 & 0.68580 \\
\hline 33 & 0.70712 & 0.46598 & 0.53998 \\
\hline 34 & 0.67398 & -0.38068 & -0.63956 \\
\hline 35 & -0.06245 & -0.50544 & 0.86696 \\
\hline 36 & -0.09577 & 0.96216 & 0.26433 \\
\hline 37 & 0.29318 & 0.79379 & 0.54237 \\
\hline 38 & -0.39873 & -0.68009 & 0.61867 \\
\hline 39 & -0.30833 & -0.07805 & 0.94937 \\
\hline 40 & -0.70424 & -0.64359 & 0.31476 \\
\hline 41 & 0.67010 & -0.17187 & -0.72841 \\
\hline 42 & -0.12359 & -0.70688 & 0.70559 \\
\hline 43 & -0.09694 & 0.64820 & -0.75729 \\
\hline 44 & -0.13014 & 0.04876 & 0.99815 \\
\hline 45 & -0.28485 & 0.86262 & -0.43728 \\
\hline 46 & 0.16745 & -0.11142 & -0.98603 \\
\hline 47 & 0.00946 & -0.00746 & 1.00873 \\
\hline 48 & 0.15374 & -0.33634 & -0.93141 \\
\hline 49 & -0.44828 & 0.85926 & -0.25853 \\
\hline 50 & -0.61673 & -0.28843 & 0.73859 \\
\hline \multicolumn{3}{|c|}{ Minimum zone error } & $7.660 \mu \mathrm{m}$ \\
\hline \multicolumn{3}{|c|}{ Least squares error } & $8.486 \mu \mathrm{m}$ \\
\hline
\end{tabular}

transition mode. In the transition mode, we need to search one more new data point to form concentric spheres having less potential energy, by the use of the proposed search scheme as described below.

The algorithm for finding the minimum zone sphericity is as follows. (a)

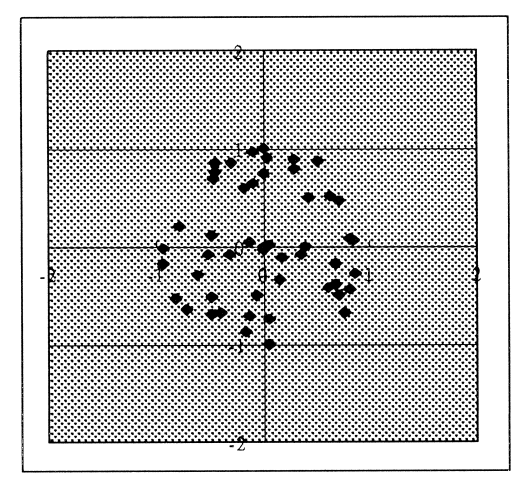

(b)

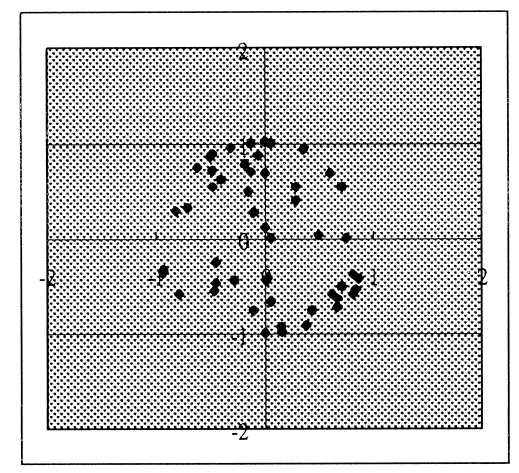

(c)

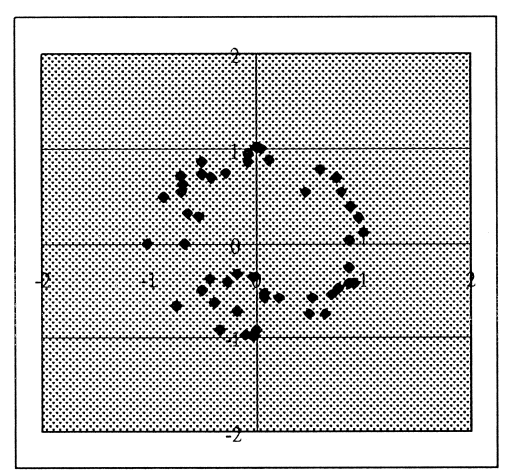

Fig. 7. Distribution of data points of Table 1 on the a) $X-Y$ plane, b) $X-Z$ plane, c) Y-Z plane.

1. Compute the least squares solution of sphericity error, and find the outer and inner enclosing spherical surfaces $C_{0}, C_{i}$ and the corresponding active data points $\mathrm{P}_{\mathrm{o}}$ and $\mathrm{P}_{\mathrm{i}}$.

2. Randomly generate two virtual data points on the inner surface $C_{i}$ to form a temporary transition mode T1-3.

3. Compute the plane equation and its normal vector formed by those three inner active points.

4. Gradually move the current sphere center along the above mentioned normal direction, up or down, as 
shown in Fig. 6a, until the first new point is contacted by the new concentric spheres determined by this point and original four active points, forming mode M2-3 or M1-4.

5. Compute the reactions of these five active points with Eq. (10) for mode M2-3, or Eq. (11) for mode M1-4, and discard one virtual point which has the most negative reaction, forming mode T1-3 or T2-2.

6. If the current mode is T1-3, repeat Steps 3 to 5 to discard the remaining virtual point.

7. If the current mode is T2-2, generate a new search line which is the intersection of two planes perpendicular to the inner line $\left(\mathrm{P}_{1} \mathrm{P}_{2}\right)$ and the outer line $\left(\mathrm{P}_{3} \mathrm{P}_{4}\right)$ respectively, as shown in Fig. 6b. A new active point will be found together with a new mode.

8. Compute the five reactions and discard the active point having the most negative reaction, and search a new point with current mode, T2-2, or T1-3, or T3-1 (similar to T1-3).

9. Repeat Step 8 until all reactions are positive.

10. The final mode must be M2-3 or M3-2, and the radial separation of the concentric spheres is the minimum zone solution.

It should be noticed here that the search scheme of this algorithm starts from the result of the least squares solution which is already very close to the minimum zone solution. The criterion of the minimum zone solution has been proven by the three Lemmas in this paper. In addition, from the geometrical point of view, we will never find two groups of concentric spheres in space both conforming to the minimum zone criterion. Therefore, the proposed algorithm can be guaranteed as the global minimum zone solution.

\section{Example}

An example shown in Table 1 is illustrated to demonstrate the criterion proposed in this research. Data points are generated randomly such that they are all located between two concentric spherical surfaces of radii equal to 0.995 and 1.005 , respectively. The distribution of data points are shown in Fig. 7 with different projection views. The result is compared with the least squares method and verified by comparing the actual spherical form error. The actual spherical form error is found by the exhausting search that examined all the possible combinations. After examining all the possible combinations for data set in Table 1, we found that the solution is the same as that in our approach. The minimum zone sphericity error is
$7.66 \mu \mathrm{m}$ dominated by the mode M3-2 with data points 7,16 , and 45 on the outer spherical surface and 20,39 on the inner one. With the use of the proposed direct search scheme we need totally only five or six iterations to get the required solution. The computer time for this example is only 1.3 seconds with a PC/486, DX-266.

\section{Conclusions}

An approach with minimum potential energy analogy to the minimum zone solution of spherical form error is proposed in this research. The problem of finding the minimum zone sphericity error is transformed into the problem of finding the minimum elastic potential energy of the corresponding mechanical system. The minimum zone solution can be justified by the proposed comprehensive criteria.

In this report, we have derived three lemmas to prove the sufficient and necessary condition of the minimum zone criteria. They could be implemented to solve the problem of minimum zone sphericity error easily. A computer program is developed which provides direct and rapid search algorithm. This concept can also be extended to solve other minimum zone form error problems. Actually, it is a unified approach for finding minimum zone form errors, such as straightness, flatness, roundness, sphericity, cylindricity, etc. A series of reports in this respect will be forthcoming.

\section{References}

[1] Cheraghi SH, Lim HS, Motavalli S. Straightness and flatness tolerance evaluation: an optimization approach. Precision Engineering 1996; 18(1):30-7.

[2] Wang Y. Minimum zone evaluation of form tolerances. Manufacturing Review 1992;5(3):213-20.

[3] Kanada T. Evaluation of spherical form errors: computation of spherical form error by means of minimum zone method and some examinations with using simulated data. Precision Engineering 1995;17(4): 281-9.

[4] Carr K, Ferreira P. Verification of form error tolerances Part I: basic issues, flatness, and straightness. Precision Engineering 1995;17(2): 131-43.

[5] Carr K, Ferreira P. Verification of form error tolerances Part II: cylindricity and straightness of a median line. Precision Engineering 1995; 17(2):144-56.

[6] Huang ST, Fan KC, Wu JH. A new minimum zone method for evaluating straightness errors. Precision Engineering 1993;15(3):158-65.

[7] Huang ST, Fan KC, Wu JH. A new minimum zone method for evaluating flatness errors. Precision Engineering 1993;15(1):25-32.

[8] Castigliano A. Theorie de l'equilibre des systemes 'elastiques et ses applications. Turin: AF Negro, 1879. 